\title{
Lipid adsorption/absorption on polycarbonate surfaces an understanding
}

\author{
CHANDRA P SHARMA and THOMAS CHANDY \\ Biomedical Technology Wing, Sree Chitra Tirunal Institute for Medical Sciences and \\ Technology, Poojapura, Trivandrum 695 012, India
}

MS received 6 January 1982; revised 7 September 1982

\begin{abstract}
Thrombus formation on a foreign surface is a complicated process, involving many factors. The adhesion and aggregation of platelets play an important role in the initial events of thrombus formation on such surfaces. In this work, adsorption/absorption studies using saturated fatty acids were carried out on polycarbonate surface to evaluate how such surfaces may affect blood-polymer interaction. The surface change was investigated by measuring the interfacial energies as derived from advancing contact angle technique. The plasma recalicification time and platelet adhesion studies were also carried out to further develop an understanding.
\end{abstract}

Keywords. Fatty acid adsorption/absorption; advancing contact angle; platelet adhesion and aggregation; plasma recalcification time; Interfacial tension; Blood compatibility; surface energy; thrombosis.

\section{Introduction}

Normal vascular endothelium is the ideal nonthrombogenic surface, which is generally considered to be resistant to platelet adhesion and aggregation. On the other hand, a foreign surface in contact with blood is thrombogenic due to platelet adhesion and aggregation. Thrombosis and coagulation induced by blood-polymer interaction are of key concern in the area of medical implants. Although several investigators have considered the nature of various polymer surfaces in the hope of developing a nonthrombogenic surface, the mechanism of thrombus formation is still not known due to the complicated nature of the process.

It has been found that concentrations of fatty acids, within the range of plasmafree fatty acid concentrations in man, can produce hypercoagulability (Warner et al 1967). Other investigators (Hoak et al 1967; Connor et al 1963; Poole 1955) indicated that fatty acids, mainly longchain saturated fatty acids, promote thrombosis via platelet aggregation mediated by ADP and accelerated the clotting by activating Hageman factor. Kim et al (1977) proposed that platelet aggregation at a polymer interface is initiated and/or accelerated by the presence of fatty acid suspension formed through the release of adsorbed fatty acid. In the paper we have attempted to correlate the surface energy parameters towards saturated fatty acid adsorption/absorption and release at the interface and the relationship with thrombosis. Platelet adhesion and plasma recalcification time studies were also carried out for a better understanding of the subject. 


\section{Experimental}

\subsection{Adsorption/absorption of fattyacids on polycarbonate}

Polycarbonate solution was prepared in dichloromethane $(10 \mathrm{mg} \% \mathrm{w} / \mathrm{v})$, films ( $\sim 0.1 \mathrm{~mm}$ thickness) cast on clean glass plates $(4.5 \times 4.5 \mathrm{~cm})$ were cut out, cleaned with $0.1 \%$ soap solution (Teepol), distilled water and finally rinsed with ethanol. The clean films were then dried in a vacuum oven at $60^{\circ} \mathrm{C}$ for $4 \mathrm{hrs}$.

The films were then exposed to saturated fatty acids of varying chainlength and a control surface in buffer. Bare surface (I set) - $0.1 \mathrm{M}$ phosphate buffer ( $\mathrm{pH} 7.4$ ). Fatty acids adsorbed/absorbed surface (II to $\mathrm{V}$ sets) $-0.1 \mathrm{M}$ fatty acid solution combined with $0.4 \mathrm{~g}$ of $\mathrm{NaOH}$ was dissolved in $0.1 \mathrm{M}$ phosphate buffer ( $\mathrm{pH} 7.4$ ). After dissolving, the solution was standardized to a $\mathrm{pH}$ of 7.4 , and made up the volume to $100 \mathrm{ml}$ with the same buffer.

Fatty acids used were butyric acid: $\mathrm{CH}_{3}-\mathrm{CH}_{2}-\mathrm{CH}_{2}-\mathrm{COOH}$ (LR, BDH Chemicals Ltd, Poole, England, $99 \%$ pure), caproic acid: $\mathrm{CH}_{3}-\left(\mathrm{CH}_{2}\right)_{4}-\mathrm{COOH}$ (LR, BDH Chemicals Ltd, Poole, England); Heptanoic acid: $\mathrm{CH}_{3}-\left(\mathrm{CH}_{2}\right)_{5}-\mathrm{COOH}$ (AR; Wilson Laboratories, Bombay) and stearic acid: $\mathrm{CH}_{3}-\left(\mathrm{CH}_{2}\right)_{16}-\mathrm{COOH}$ (AR, Wilson Laboratories, Bombay). Dissolving stearic acid completely was difficult, however since the adsorption/absorption is in $\mu \mathrm{g}$ levels, the experimental data were used to study relative variations.

The films were kept in the respective fatty acid solutions for varying time intervals, taken out; rinsed with phosphate buffer under controlled flow rate to remove excess fatty acids on the surface. The films were dried overnight in vacuum oven at room temperature and the contact angle'studies were carried out using a goniometer (Kernco Instruments, Texas) and platelet adhesion.

Surface energy parameters of various fatty acid adsorbed/absorbed surfaces were obtained from advancing contact angle technique. The contact angles for each set of film were measured using a goniometer and spectroscopically pure solvents. From the contact angle, the values like $\gamma_{\mathrm{SV}}$ and $\gamma_{\mathrm{SL}}$ were obtained from the conversion tables of Neumann et al (1980). Contact angle experiments were repeated at different lengths of time (short time exposures in minutes of $10,20,30,40,60,80$ and 120 and long terms of 5, 20,35,50 and 70 days): however no significant changes were observed with time of exposure and molecular length of the fattyacid. Therefore the data for $10 \mathrm{~min}$ exposure to butyric acid alone has been presented in table 1 .

\subsection{Platelet adhesion on fattyacid adsorbed/absorbed surfaces}

Washed human platelets were prepared as described (Sharma and Chandy 1982) and suspended in tyrode solution (Lee and Kim 1979) for the adhesion studies.

The vacuum-dried films were exposed to platelet suspension for $1 \mathrm{hr}$ in a dustfree area, rinsed with buffer of $\mathrm{pH} 7.4$, under controlled flow rate for $3 \mathrm{~min}$, and the platelets fixed with $2.5 \%$ glutaraldehyde [ $(50 \% \mathrm{w} / \mathrm{v})$ Koch-Light Laboratories,

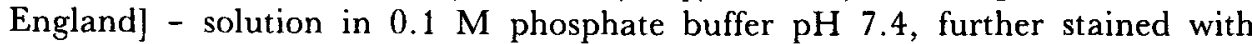
Coomassie Blue $\mathrm{G}$. The platelet density was detected using an optical microscope. Different vision fields were read randomly and averaged in similar fashion for all samples. 
Table 1. Surface energy components on butyric acid exposed polycarbonate films (10 min exposure).

\begin{tabular}{|c|c|c|c|c|c|c|c|}
\hline \multirow{2}{*}{ Liquids } & \multirow{2}{*}{$\gamma_{L V}^{b}$} & \multicolumn{3}{|c|}{ Bare Surface } & \multicolumn{3}{|c|}{ Butyric acid adsorbed surface } \\
\hline & & $\theta^{\mathbf{a}}$ & $\gamma_{\mathrm{SV}}^{\mathrm{b}}$ & $\gamma_{\mathrm{SL}}^{\mathrm{b}}$ & $\theta^{\mathrm{a}}$ & $\gamma_{\mathrm{SV}}^{\mathrm{b}}$ & $\gamma_{\mathrm{SL}}^{\mathbf{b}}$ \\
\hline Water & 72.5 & $70 \pm 1$ & 41.2 & 16.4 & $72.5 \pm 1$ & 39.7 & 17.9 \\
\hline Glycerol & 63.0 & $62 \pm 1$ & 38.3 & 8.7 & $64 \pm 1$ & 37.2 & 9.5 \\
\hline Formamide & 58.0 & $54 \pm 1$ & 39.0 & 4.9 & $55 \pm 1$ & 38.5 & 5.2 \\
\hline $\begin{array}{l}\text { Ethylene- } \\
\text { glycol }\end{array}$ & 47.7 & $44 \pm 1.5$ & 36.3 & 1.8 & $46 \pm 1$ & 35.4 & 2.1 \\
\hline Mean & $\mathrm{b}=$ & $38.7 \pm 2.5$ & & & $\begin{array}{r}\mathrm{Me} \\
\text { atact angle }\end{array}$ & $\begin{array}{l}\mathrm{b} \\
\mathrm{SV}= \\
\mathrm{S} . \mathrm{D} .\end{array}$ & $\begin{array}{l}9 \pm 2 \\
\text { ergs/c }\end{array}$ \\
\hline
\end{tabular}

\subsection{Coagulation studies}

2.3a Plasma recalcification time: Citrated human plasma was collected and tested for recalcification time under controlled $\mathrm{pH}$ and temperature with various saturated fatty acid adsorbed/absorbed polymer surface.

Glass tubes (dimension $10 \times 75 \mathrm{~mm}$ ) were coated with $10 \%$ polycarbonate and were dipped in $0.1 \mathrm{M}$ fatty acids (as described earlier) for varying time intervals. After adsorption/absorption with time, the tubes were taken out and rinsed carefully with buffer to remove excess fatty acids. The tubes were then dried overnight in vacuum oven at room temperature. Control PC-coated tubes were also prepared.

The clotting time was determined as described by Austen, and Rhymes (1975). The test was repeated until reproducibility was assured.

\section{Discussion}

It is proposed that the presence of saturated fattyacids (within the range of plasmafree fattyacid concentrations in humans) can produce hypercoagulability (Warner et al 1967) and can promote thrombosis (Hoak et al 1967; Prost-Dvojakovic and Samma 1973). Our experimental observations basically support that fattyacid adsorption/absorption on a polymer surface has adverse effects towards blood compatibility, since the platelet adhesion is increased and there is a decrease in plasma recalcification time (PRT) relatively compared to bare surface (table 2). Further interfacial tension of lipid adsorbed/absorbed surface is also increased probably due to encouraged hydrophobic behaviour of the surface (table 1). So it seems that the surface properties of the material play an important role for other blood components to adhere to the surface, which may again enhance the chances of thrombus formation.

Although, no significant variations are observed with exposure time and increase in molecular length from butyric acid to stearic acid in our contact angle data, it 
Table 2. Platelet adhesion and plasma recalcification time on fattyacids exposed polycarbonate films (10 min. exposure)

\begin{tabular}{lcc}
\hline Surfaces & $\begin{array}{c}\text { No. of platelets } \\
\text { adhered to fatty acids } \\
\text { adsorbed surfaces } \pm \\
\text { S.D. }\end{array}$ & PRT in seconds \pm S.D. \\
\hline Bare & $6 \pm 1$ & $148.5 \pm 1.5$ \\
Butyric acid & $9 \pm 2$ & $137 \quad \pm 6$ \\
Caproic acid & $9 \pm 2$ & $126 \quad \pm 1.5$ \\
Heptanoic acid & $9 \pm 2$ & $128 \quad \pm 3$ \\
Stearic acid & $10 \pm 3$ & $128 \quad \pm 3$ \\
\hline
\end{tabular}

appears that it encourages the process of platelet adhesion and decreases the plasma recalcification time. This shows that the initial events of lipid adsorption/absorption on a polymer surface is extremely important towards the process of thrombosis.

It has been suggested (Kim et al 1977) that the fattyacid suspension may result in a high $\mathrm{Ca}^{2}+$ concentration at the platelet-extracellular region and subsequently increased biochemical release from the cells. Also, negatively-charged colloidal particles of fatty acid (micelle) may accelerate the platelet aggregation and clotting. This supports our observations, since a number of platelet aggregates were present on the polymer surface precoated with excess fatty acids. Also, there was a decrease in PRT. So we feel that the increased $\mathrm{Ca}^{2}+$ ion concentration and the negative charge of the micellar lipid alter the platelet function towards encouraged adhesion of the cells and triggering of the coagulation cascade system.

The charge of the micelles will influence the possibilities for both $\mathrm{Ca}^{2}+$ ions and charged proteins to approach the micelle and attach to its surface by electrostatic forces (Kahn and Kemker 1969). Apart from charge, hydrophobicity of the micelle also helps in determining its coagulation activity. Our surface energy and contact angle data suggest an increase in interfacial tension and a decrease in surface energy on fatty acid adsorbed/absorbed surfaces, which clearly indicates the hydrophobic character of the surface and the possible increase in interactions at the blood-polymer interface.

It is obvious that (Harry P Gregor 1975) electrostatic forces are only one of a number of noncovalent interactions and that it is the overall balance of these forces including hydrogen bonds, electrostatic forces, Van der Waals London dispersion forces and hydrophobic "forces" that determines not only the structure of micelles and polymers but also the interactions that occur between them. In addition to its importance in determining structure, hydrophobic effects are also the primary driving force involved in micelle-polymer interaction. So the possible interaction of lipids with polycarbonate can be demonstrated as follows. Both polycarbonate and micellar lipid are amphipathic in nature, i.e. each contains both hydrophilic and hydrophobic moieties due to polar and nonpolar groups distributed on the surface. 
Adsorption/absorption of lipids on to polycarbonate take place satisfying the affinities of relative polar and nonpolar groups of both interacting molecules. During this process of adsorption/absorption net variation of surface energy parameters have not been much significant, however the slight shift observed seems to be towards the increase in the hydrophobic behaviour of the surface. Such a net hydrophobic effect and the variations caused by the chemical entities of the lipid adsorbed/absorbed surface, encourages the platelet adhesion and reduces the PRT, which ultimately causes clotting.

\section{Acknowledgements}

We appreciate the help received from the Blood Bank Group of the Institute for providing the blood. Thanks are also due to Miss M K Sheela for assistance.

\section{References}

Austen D E G and Rhymes I L 1975 A laboratory manual of blood coagulation (Oxford: Blackwells) p. 35 Connor W E, Hoark J C and Warner E D $1963 \mathrm{~J}$. Clin. Invest. 42860

Harry P Gregor 1975 Biomedical applications of polymers (New York and London: Plenum) p. 19

Hoak J C, Warner E D and Connor W E 1967 Circ. Res. 2011

Kahn M J P and Hember H C 1969 Thrombos. Diathes. Haemorth. 22417

Kim S W, Wisniewski S, Lee E S and Winn M L 1977 J. Biomed. Mater. Res. Symp. No. 823

Lee E S and Kin S W 1979 Trans. Am. Soc. Artif. Int. Organs 25124

Neumann A W, Absolom D R, Francis D W and Vanoss C J 1980 Separation and purification methods 969

Poole J C F 1955 Br.J. Exp. Pathol. 36248

Prost-Drojakovic R J and Samma M 1973 Haemostatis 273

Sharma C P and Chandy T 1982 J. Colloid. Interface Sci. 89479

Warner E D, Hoak J C and Connor W E 1967 Thromb. Diath. Haemorr. Suppl. 26249 